\title{
Study of Wireless Sensor Network
}

\author{
Mohaiminul Islam ${ }^{1}$, Amit Kumar ${ }^{2}$, Anower Hossain ${ }^{3}$ \\ ${ }^{1}$ School of Intelligent Technology and Engineering, Chongqing University of Science and Technology, Chongqing, China \\ ${ }^{2}$ School of Electrical Engineering and Information Technology, Chongqing University of Science and Technology, Chongqing, China \\ ${ }^{3}$ School of Computer Science and Engineering, Chongqing University of Technology, Chongqing, China
}

Email address:

mohaiminu100@hotmail.com (M. Islam), amitbiswas0082@gmail.com (A. Kumar), anowercse33@gmail.com (A. Hossain)

\section{To cite this article:}

Mohaiminul Islam, Amit Kumar, Anower Hossain. Study of Wireless Sensor Network. International Journal of Sensors and Sensor Networks. Vol. 7, No. 1, 2019, pp. 9-15. doi: 10.11648/j.jijssn.20190701.12

Received: July 27, 2019; Accepted: August 18, 2019; Published: August 26, 2019

\begin{abstract}
A wireless sensor network is a group of specialized transducers with a communications infrastructure for monitoring and recording conditions at diverse locations. Commonly monitored parameters are temperature, humidity, pressure, wind direction and speed, illumination intensity, vibration intensity, sound intensity, power-line voltage, chemical concentrations, pollutant levels and vital body functions. A sensor network consists of multiple detection stations called sensor nodes, each of which is small, lightweight and portable. Every sensor node is equipped with a transducer, microcomputer, transceiver and power source. The transducer generates electrical signals based on sensed physical effects and phenomena. The microcomputer processes and stores the sensor output. The transceiver receives commands from a central computer and transmits data to that computer. The power for each sensor node is derived from a battery. Currently, WSN (Wireless Sensor Network) is the most standard services employed in commercial and industrial applications, because of its technical development in a processor, communication, and low-power usage of embedded computing devices. The WSN is built with nodes that are used to observe the surroundings like temperature, humidity, pressure, position, vibration, sound etc. These nodes can be used in various real-time applications to perform various tasks like smart detecting, a discovery of neighbor node, data processing and storage, data collection, target tracking, monitor and controlling, synchronization, node localization, and effective routing between the base station and nodes. This paper describes the concept of wireless sensor network.
\end{abstract}

Keywords: Wireless Sensor Network, Protocols Sensor, Network Topologies, Application Layer, Data Transmission, Ad Hoc Networks, Network Architecture

\section{Introduction}

WSNs were initially designed to facilitate military operations but its application has since been extended to health, traffic, and many other consumer and industrial areas. A WSN consists of anywhere from a few hundreds to thousands of sensor nodes. The sensor node equipment includes a radio transceiver along with an antenna, a microcontroller, an interfacing electronic circuit, and an energy source, usually a battery. The size of the sensor nodes can also range from the size of a shoe box to as small as the size of a grain of dust. As such, their prices also vary from a few pennies to hundreds of dollars depending on the functionality parameters of a sensor like energy consumption, computational speed rate, bandwidth, and memory [1].

A Wireless Sensor Network is one kind of wireless network includes a large number of circulating, self-directed, minute, low powered devices named sensor nodes called motes. These networks certainly cover a huge number of spatially distributed, little, battery-operated, embedded devices that are networked to caringly collect, process, and transfer data to the operators, and it has controlled the capabilities of computing $\&$ processing. Nodes are the tiny computers, which work jointly to form the networks [2-3].

The sensor node is a multifunctional, energy efficient wireless device. The applications of motes in industrial are widespread. A collection of sensor nodes collects the data from the surroundings to achieve specific application objectives. The communication between motes can be done with each other using transceivers. In a wireless sensor network, the number of motes can be in the order of hundreds/ even thousands. In contrast with sensor n/ws, Ad 
Hoc networks will have fewer nodes without any structure.

\section{Wireless Sensor Network Architecture}

The most common WSN architecture follows the OSI architecture Model. The architecture of the WSN includes five layers and three cross layers. Mostly in sensor $n / w$ we require five layers, namely application, transport, $\mathrm{n} / \mathrm{w}$, data link \& physical layer. The three cross planes are namely power management, mobility management, and task management. These layers of the WSN are used to accomplish the $\mathrm{n} / \mathrm{w}$ and make the sensors work together in order to raise the complete efficiency of the network [4].

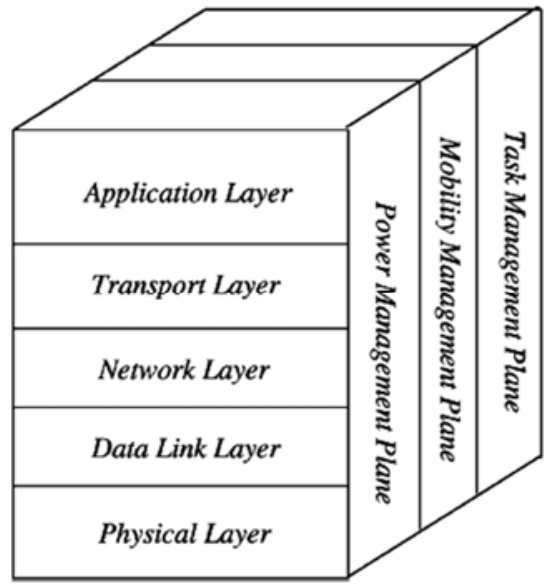

Figure 1. Layer of wireless sensor networks

\subsection{Application Layer}

The application layer is liable for traffic management and offers software for numerous applications that convert the data in a clear form to find positive information. Sensor networks arranged in numerous applications in different fields such as agricultural, military, environment, medical, etc.

\subsection{Transport Layer}

The function of the transport layer is to deliver congestion avoidance and reliability where a lot of protocols intended to offer this function are either practical on the upstream. These protocols use dissimilar mechanisms for loss recognition and loss recovery. The transport layer is exactly needed when a system is planned to contact other networks [5].

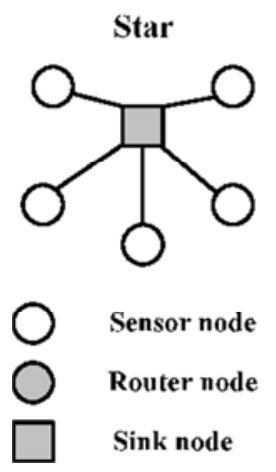

Providing a reliable loss recovery is more energy efficient and that is one of the main reasons why TCP is not fit for WSN. In general, Transport layers can be separated into Packet driven, Event driven. There are some popular protocols in the transport layer namely STCP (Sensor Transmission Control Protocol), PORT (Price-Oriented Reliable Transport Protocol and PSFQ (pump slow fetch quick).

\subsection{Network Layer}

The main function of the network layer is routing, it has a lot of tasks based on the application, but actually, the main tasks are in the power conserving, partial memory, buffers, and sensor don't have a universal ID and have to be self-organized.

The simple idea of the routing protocol is to explain a reliable lane and redundant lanes, according to a convinced scale called metric, which varies from protocol to protocol. There are a lot of existing protocols for this network layer, they can be separate into; flat routing and hierarchal routing or can be separated into time driven, query-driven \& event driven [6].

\subsection{Data Link Layer}

The data link layer is liable for multiplexing data frame detection, data streams, MAC, \& error control, confirm the reliability of point-point (or) point-multipoint.

\subsection{Physical Layer}

The physical layer provides an edge for transferring a stream of bits above physical medium. This layer is responsible for the selection of frequency, generation of a carrier frequency, signal detection, Modulation \& data encryption. IEEE 802.15.4 is suggested as typical for low rate particular areas \& wireless sensor network with low cost, power consumption, density, the range of communication to improve the battery life. CSMA/CA is used to support star \& peer to peer topology. There are several versions of IEEE 802.15.4. V [7].

\section{WSN Network Topologies}

For radio communication networks, the structure of a WSN includes various topologies like the ones given below.
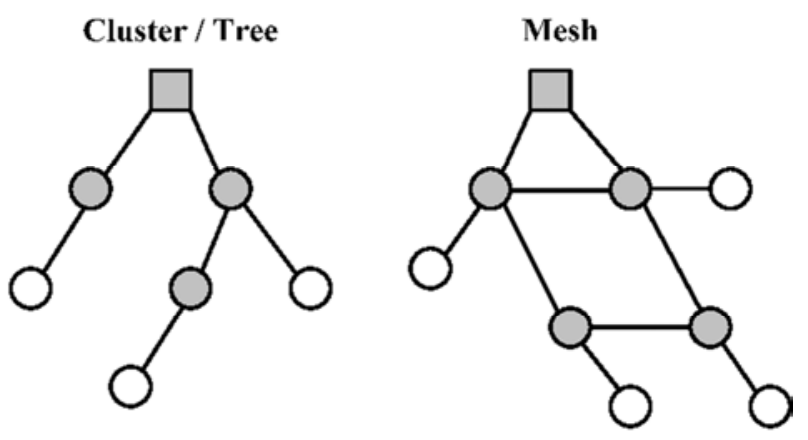

Figure 2. Wireless Sensor Network Topologies. 


\subsection{Star Topologies}

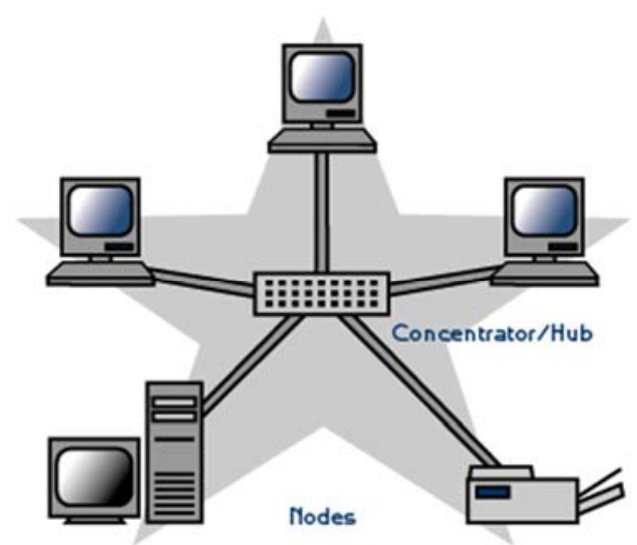

Figure 3. Star Topologies.

Star topology is a communication topology, where each node connects directly to a gateway. A single gateway can send or receive a message to a number of remote nodes. In star topologies, the nodes are not permitted to send messages to each other. This allows low-latency communications between the remote node and the gateway (base station).

Due to its dependency on a single node to manage the network, the gateway must be within the radio transmission range of all the individual nodes. The advantage includes the ability to keep the remote nodes' power consumption to a minimum and simply under control. The size of the network depends on the number of connections made to the hub [8].

\subsection{Tree Topologies}

Tree topology is also called as cascaded star topology. In tree topologies, each node connects to a node that is placed higher in the tree, and then to the gateway. The main advantage of the tree topology is that the expansion of a network can be easily possible, and also error detection becomes easy. The disadvantage with this network is that it relies heavily on the bus cable; if it breaks, all the network will collapse.

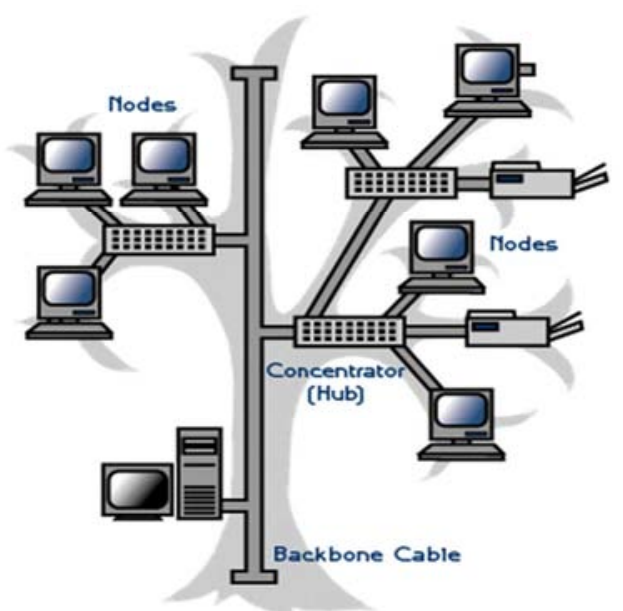

Figure 4. Tree Network Topologies.

\subsection{Mesh Topologies}

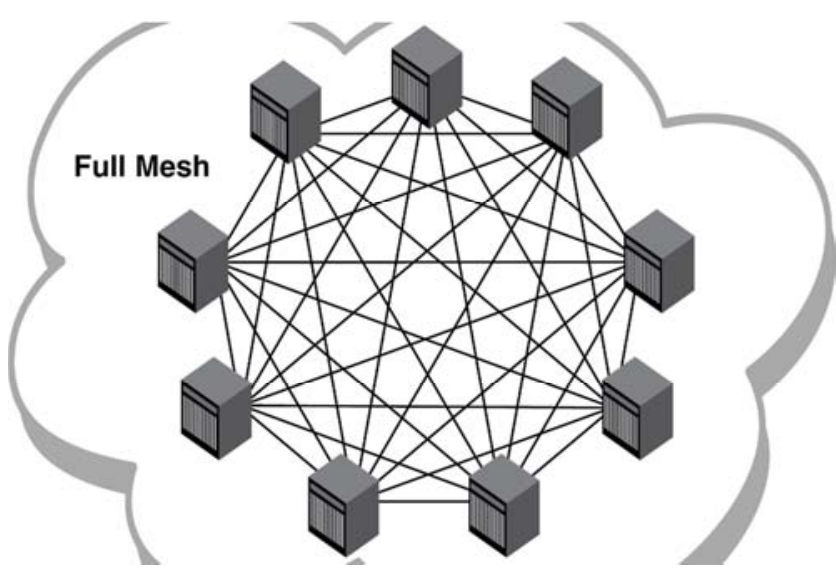

Figure 5. Mesh Topologies.

The Mesh topologies allow transmission of data from one node to another, which is within its radio transmission range. If a node wants to send a message to another node, which is out of radio communication range, it needs an intermediate node to forward the message to the desired node [9]. The advantage with this mesh topology includes easy isolation and detection of faults in the network. The disadvantage is that the network is large and requires huge investment.

\section{Types of WSNs (Wireless Sensor Networks)}

Depending on the environment, the types of networks are decided so that those can be deployed underwater, underground, on land, and so on. Different types of WSNs include:

1. Terrestrial WSNs.

2. Underground WSNs.

3. Underwater WSNs.

4. Multimedia WSNs.

5. Mobile WSNs.

\subsection{Terrestrial WSNs}

Terrestrial WSNs are capable of communicating base stations efficiently, and consist of hundreds to thousands of wireless sensor nodes deployed either in unstructured (ad hoc) or structured (Preplanned) manner. In an unstructured mode, the sensor nodes are randomly distributed within the target area that is dropped from a fixed plane. The preplanned or structured mode considers optimal placement, grid placement, and 2D, 3D placement models [10].

In this WSN, the battery power is limited; however, the battery is equipped with solar cells as a secondary power source. The Energy conservation of these WSNs is achieved by using low duty cycle operations, minimizing delays, and optimal routing, and so on. 


\subsection{Underground WSNs}

The underground wireless sensor networks are more expensive than the terrestrial WSNs in terms of deployment, maintenance, and equipment cost considerations and careful planning. The WSNs networks consist of a number of sensor nodes that are hidden in the ground to monitor underground conditions. To relay information from the sensor nodes to the base station, additional sink nodes are located above the ground.
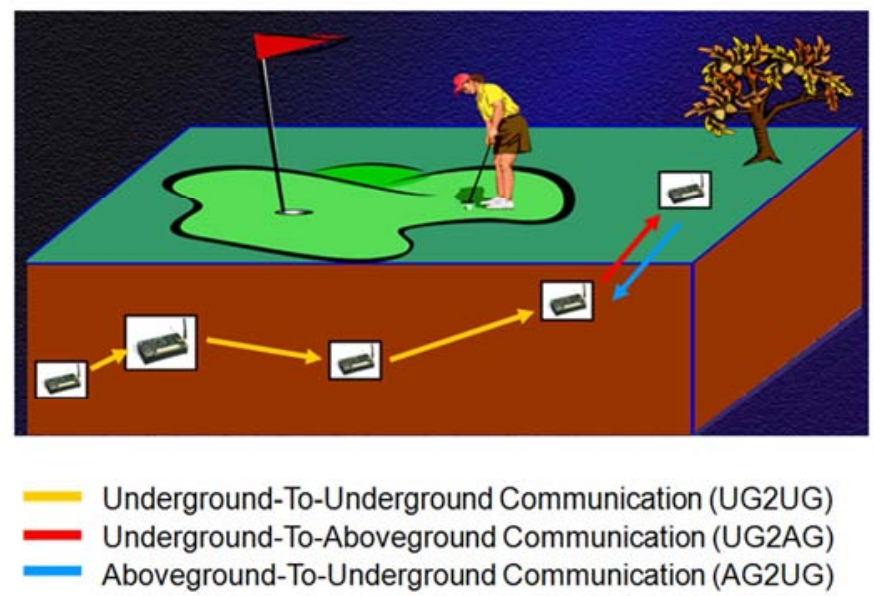

Figure 6. Underground wireless sensor networks.

The underground wireless sensor networks deployed into the ground are difficult to recharge. The sensor battery nodes equipped with a limited battery power are difficult to recharge.
In addition to this, the underground environment makes wireless communication a challenge due to high level of attenuation and signal loss.

\subsection{Under Water WSNs}

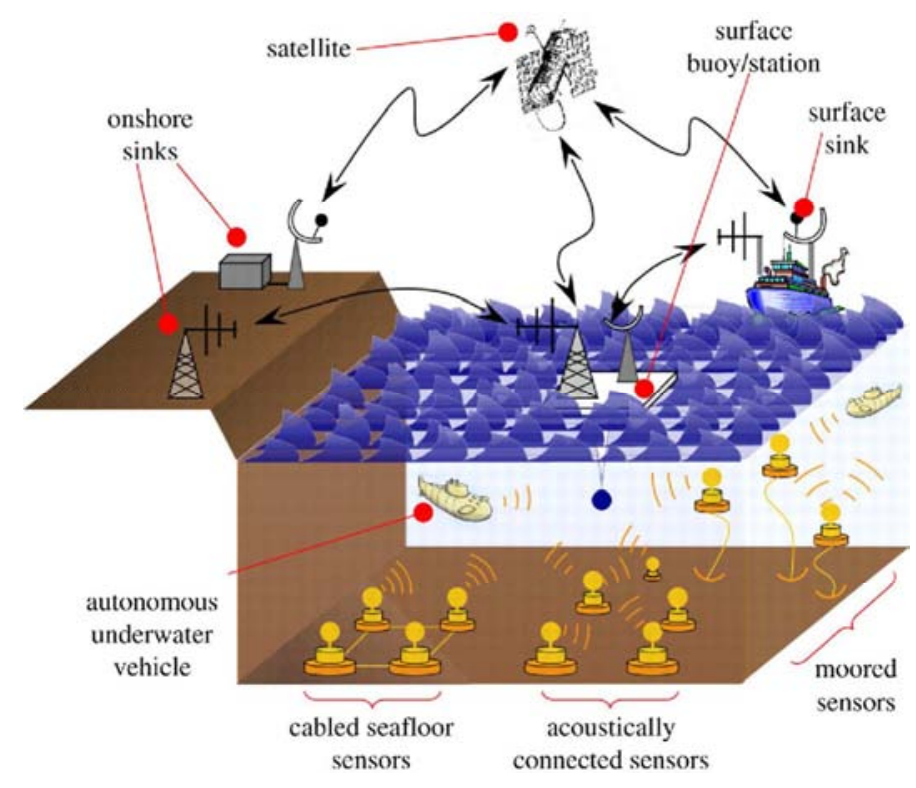

Figure 7. Underwater acoustic sensor networks.

More than $70 \%$ of the earth is occupied with water. These networks consist of a number of sensor nodes and vehicles deployed under water. Autonomous underwater vehicles are used for gathering data from these sensor nodes. A challenge of underwater communication is a long propagation delay, and bandwidth and sensor failures.

Under water WSNs are equipped with a limited battery that cannot be recharged or replaced. The issue of energy conservation for under water WSNs involves the development of underwater communication and networking techniques.

\subsection{Multimedia WSNS}

Muttimedia wireless sensor networks have been proposed to enable tracking and monitoring of events in the form of multimedia, such as imaging, video, and audio. These networks consist of low-cost sensor nodes equipped with micrpphones and cameras. These nodes are interconnected 
with each other over a wireless connection for data compression, data retrieval and correlation.

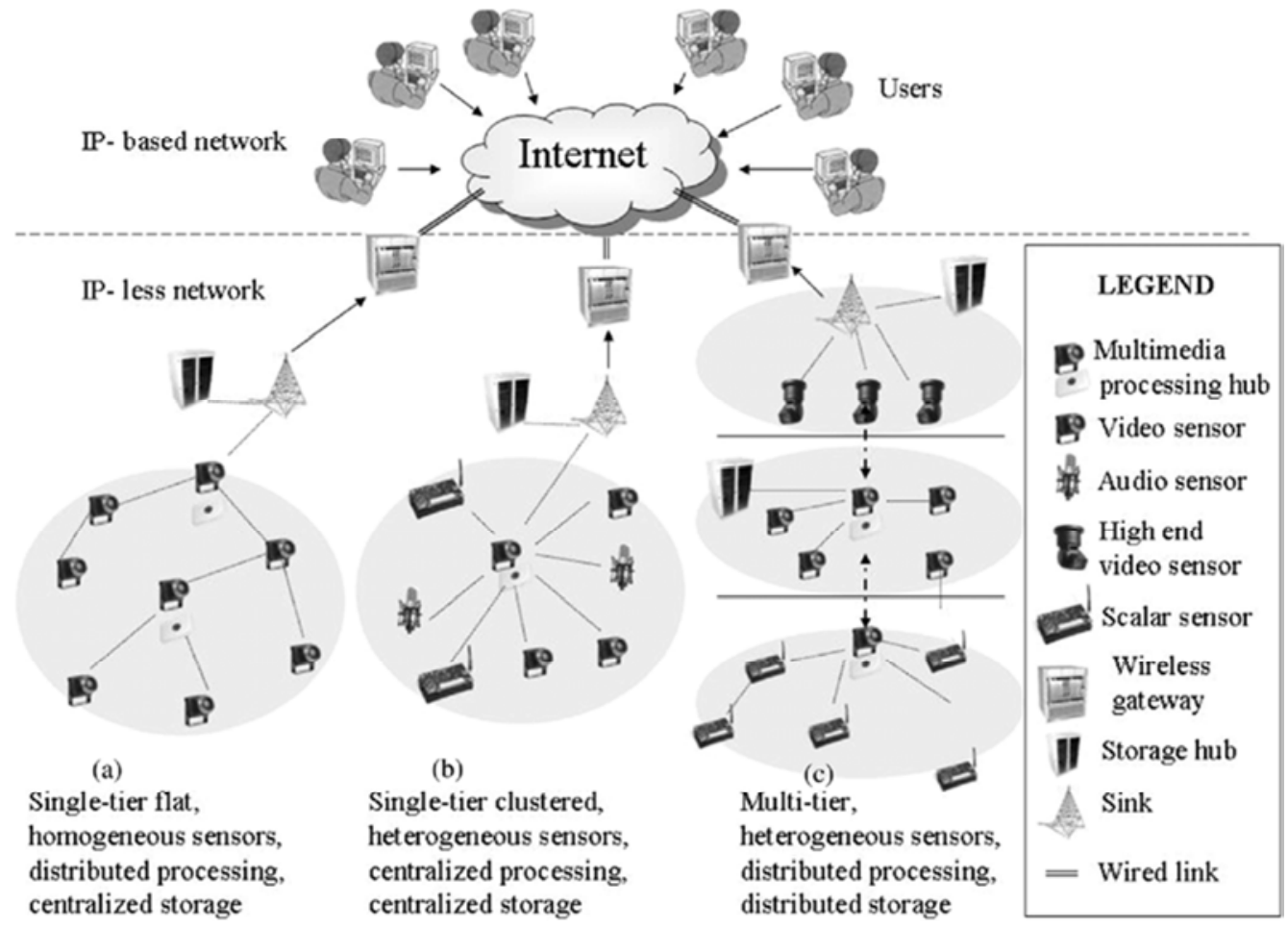

Figure 8. Multimedia wireless sensor networks.

The challenges with the multimedia WSN include high energy consumption, high bandwidth requirements, data processing and compressing techniques. In addition to this, multimedia contents require high bandwidth for the contents to be delivered properly and easily [11].

\subsection{Mobile WSNs}

These networks consist of a collection of sensor nodes that can be moved on their own and can be interacted with the physical environment. The mobile nodes have the ability to compute sense and communicate.

The mobile wireless sensor networks are much more versatile than the static sensor networks. The advantages of MWSN over the static wireless sensor networks include better and improved coverage, better energy efficiency, superior channel capacity, and so on.

\section{Wireless Sensor Networks Applications}

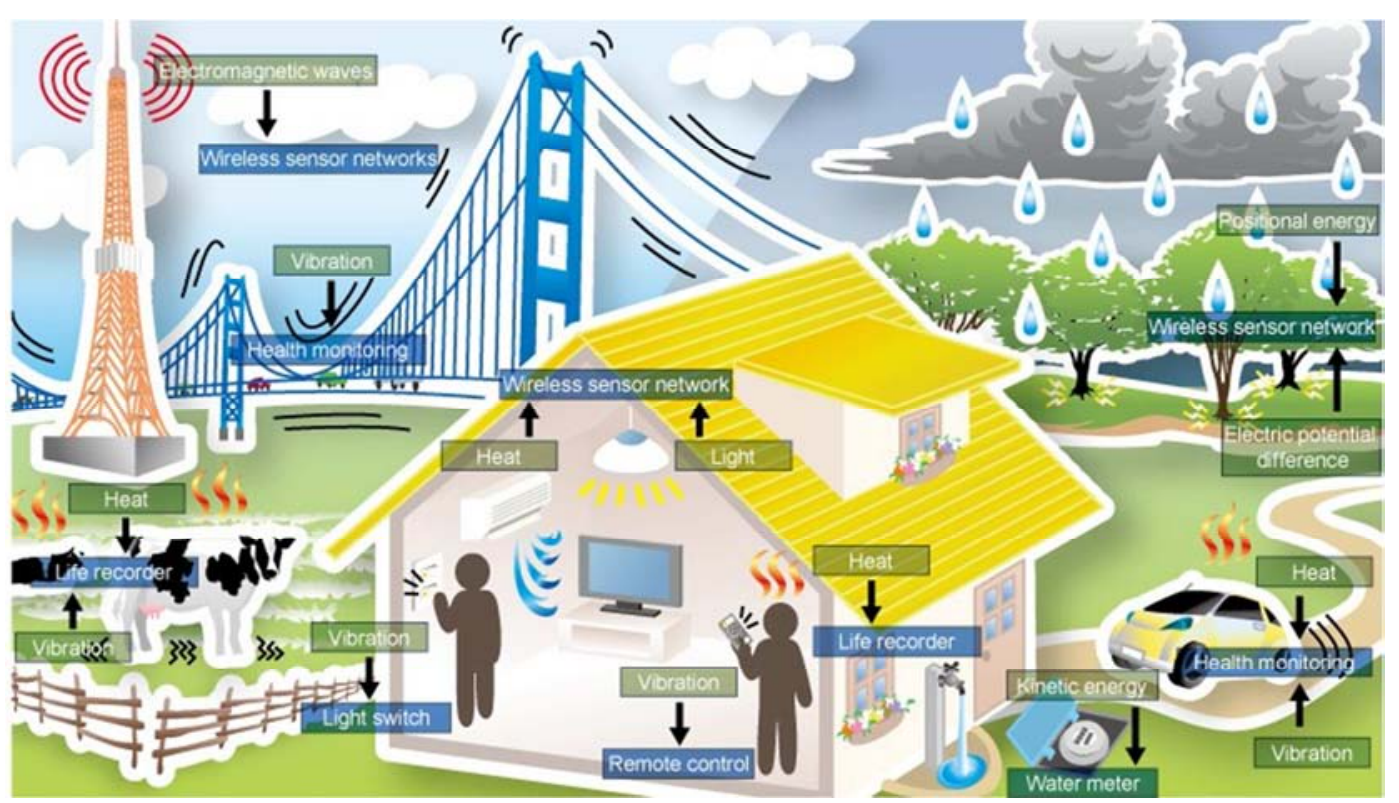

Figure 9. Wireless Sensor Networks Applications. 
a) These networks are used in environmental tracking, such as forest detection, animal tracking, flood detection, forecasting and weather prediction, and also in commercial applications like seismic activities prediction and monitoring.

b) Military applications, such as tracking and environment monitoring surveillance applications use these networks. The sensor nodes from sensor networks are dropped to the field of interest and are remotely controlled by a user. Enemy tracking, security detections are also performed by using these networks.

c) Health applications, such as Tracking and monitoring of patients and doctors use these networks.

d) The most frequently used wireless sensor networks applications in the field of Transport systems such as monitoring of traffic, dynamic routing management and monitoring of parking lots, etc., use these networks.

e) Rapid emergency response, industrial process monitoring, automated building climate control, ecosystem and habitat monitoring, civil structural health monitoring, etc., use these networks [12].

\section{Characteristics of Wireless Sensor Network}

The characteristics of WSN include the following:

a) The consumption of Power limits for nodes with batteries.

b) Capacity to handle with node failures.

c) Some mobility of nodes and Heterogeneity of nodes.

d) Scalability to large scale of distribution.

e) Capability to ensure strict environmental conditions.

f) Simple to use.

g) Cross-layer design.

\section{Advantages of Wireless Sensor Networks}

The advantages of WSN include the following:

a) Network arrangements can be carried out without immovable infrastructure.

b) Apt for the non-reachable places like mountains, over the sea, rural areas and deep forests.

c) Flexible if there is a casual situation when an additional workstation is required.

d) Execution pricing is inexpensive.

e) It avoids plenty of wiring.

f) It might provide accommodations for the new devices at any time.

g) It can be opened by using a centralized monitoring [13, 14].

\section{Limitations of Wireless Sensor Networks}

a) Possess very little storage capacity - a few hundred kilobytes.

b) Possess modest processing power- $8 \mathrm{MHz}$.

c) Works in short communication range - consumes a lot of power.

d) Requires minimal energy - constrains protocols.

e) Have batteries with a finite life time.

f) Passive devices provide little energy [15].

\section{Conclusion}

Smart environments represent the next evolutionary development step in building, utilities, industrial, home, shipboard, and transportation systems automation. Like any sentient organism, the smart environment relies first and foremost on sensory data from the real world. Sensory data comes from multiple sensors of different moralities in distributed locations. The smart environment needs information about its surroundings as well as about its internal workings; this is captured in biological systems by the distinction between exteroceptors and proprioceptors. Wireless sensor networks are a group of specialized devices or sensors which are used to monitor different environmental conditions and to collect and organize that data at some certain central location. It detects and measures a number of physical conditions such as humidity, temperature, sound, pressure, speed and direction, chemical concentrations, vibrations, pollutant levels and many other such conditions. It has much application with microcontroller projects. Nowadays wireless sensor network is playing a important role in the whole world.

\section{References}

[1] E. Altman, T. Basar, T. Jimenez, and N. Shimkin, "Competitive routing in networks with polynomial costs," IEEE Trans. Automat. Control, vol. 47, no. 1, pp. 92-96, 2002.

[2] R. Bronson and G. Naadimuthu, Operations Research, 2 ed., Schaum's Outlines, McGraw Hill, New York, 1997.

[3] N. Swamy, Control Algorithms for Networked Control and Communication Systems, PhD Thesis, Dept. of Elect. Eng, The University of Texas at Arlington, Texas, 2003.

[4] J. W. Smith, Rand Corp., On Distributed Communications, Memorandum RM-3578-PR, 1964, http://www.rand.org/publications/RM/RM3578/.

[5] S. Shah-Heydari and O. Yang, "A tree-based algorithm for protection/restoration in optical mesh networks," Proc. Canadian Conf. Elect. and Comp. Eng., vol. 2, pp. 1169-1174, Canada 2001. 
[6] J. Shi and J. P. Fonseka, "Hierarchical self-healing rings," IEEE/ACM Trans. Networking, vol. 3, no. 6, pp. 690-697, Dec. 1995. Smart Transducer Interface Standard, IEEE 1451, Sensors Expo, Philadelphia, Oct. 2001.

[7] S. Ray, "An introduction to ultra wide band (impulse) radio," Internal Report, Elect. and Computer Eng. Dept, Boston Univ., Oct. 2001.

[8] D. M. Rudkevich, J. Scheerder, and D. N. Reinhoudt, "Anion recognition by natural receptors," inMolecular Design and Bioorganic Catalysis, ed. C. S. Wilcox, pp. 137-162, Kluwer, Boston, 1996.

[9] K. Liu, M. Fitzgerald, and F. L. Lewis, "Kinematic analysis of a Stewart Platform manipulator," IEEE Trans. Industrial Electronics, vol. 40, no. 2, pp. 282-293, 1993.

[10] E. S. Kolesar, C. P. Brothers, C. P. Howe, et al., "Integrated circuit microsensor for selectively detecting nitrogen dioxide and diisopropyl methylphosphate," Thin Solid Films, vol. 220, pp. 30-37. 1992.

[11] G. T. A. Kovacs, Micromachined Transducers Sourcebook, McGraw-Hill, Boston, 1998.

[12] P. R. Kumar, "New technological vistas for systems and control: the example of wireless networks," IEEE Control Systems Magazine, pp. 24-37, Feb. 2001.

[13] F. L. Lewis, Optimal Estimation, Wiley, New York, 1986.

[14] F. L. Lewis, Applied Optimal Control and Estimation, Prentice-Hell, New Jersey, 1992.

[15] EEE 1451, A Standard Smart Transducer Interface, Sensors Expo, Philadelphia, Oct. 2001, http://ieee1451.nist.gov/Workshop_04Oct01/1451_overview.pdf. 